\title{
Failure Mode Analysis of CORBA Service Implementations
}

\author{
Eric Marsden and Jean-Charles Fabre \\ LAAS-CNRS, Toulouse, France \\ \{emarsden, fabre\}@laas.fr
}

\begin{abstract}
Before using middleware in critical systems, integrators need information on the robustness of the software. This includes having a clear idea of the failure modes of middleware candidates, including their core and side elements. In this paper we describe ongoing work on the failure mode analysis of CORBA-based middleware. Our initial work targets the CORBA Name Service, and the characterization is addressed using fault injection techniques. We present the results of injecting corrupted messages at the targeted middleware. Our experiments have been performed on four different implementations, and some comparisons are provided. First lessons learnt from these experiments, from a critical system integrator's viewpoint, are also reported.
\end{abstract}

\section{Introduction}

Middleware is being applied to an increasingly wide range of application domains, including critical applications such as Air Traffic Control systems, electronic interlocking systems in railways, and space platforms. In these application domains, dependability issues are of prime importance. The failure of these systems may put human lives at risk, or cause significant economic losses. However, there has been little published work on the dependability characterization of middleware. Information on the robustness of middleware implementations is useful from two points of view:

- Assisting system integrators in selecting the middleware candidate that is best suited to their requirements;

- Aiding middleware implementers by providing data on vulnerable points of their software, which may suggest alternative design or implementation techniques.

The analysis of system behaviour in the presence of faults is a crucial and complex issue. Faults can impact all system layers, and their effect may propagate from one layer to the other. The failure modes of a middleware can thus be very much dependent on the failure modes of the underlying operating system. In addition, the behaviour of the system depends on the types of faults to which it is exposed. These encompass design and physical faults. The interest of the results that can be obtained rely on the fault assumptions which are made 
for the experiments, and on the assumptions made concerning the behaviour of companion components in the system.

The long-term objective of this work is to address multiple facets of the characterization of a middleware implementation. Our first work presented here focuses on the characterization of the CORBA Naming Service, which constitutes a single point of failure in most CORBA-based systems.

The paper is organized as follows. In Section [2] we introduce terminology from the dependability community, and present the experimental dependability characterization technique that we have applied to the CORBA Naming Service. In Section 3. we describe the overall methodology, including the fault assumptions, the classification of the possible failure modes, and our experimental setup. We then summarize the experimental results obtained in Section 4, and discuss related work in Section 5. Section [6 presents some first lessons which can be learned from our work, from the viewpoint of a system integrator, and we draw the conclusions in Section 7

\section{Dependability and Failure Modes}

The dependability of a system is defined as the ability to place a justified reliance in the service it delivers 1 . When a system no longer delivers the service that is expected of it, it is said to have failed. Failure occurs at the interface of the system with its environment, and results from the perception of an erroneous internal state. The hypothesized origin of this error is called a fault. Thus, there is a causal chain from fault to error to failure.

Users of a component need to reach a certain level of confidence in its ability correctly to deliver service. One process towards reaching this confidence is the analysis of the component's failure modes. An understanding of a component's behaviour in the presence of faults can be used by system integrators to decide on the mechanisms to be used to tolerate the remaining faults.

There are two main approaches to obtaining information on a component's failure modes: (i) analysis of error logs from a large number of operational systems, as in [2], or (ii) by simulating faults and observing the resulting behaviour. The first approach relies on error information obtained either from logs maintained by system administrators or from automatic surveillance mechanisms provided by the system. The former are often subjective, incomplete and not always available. The latter generally provide more structured information and thus are more appropriate for later analysis. However, faulty conditions are rare and thus obtaining a sufficient set of data is problematic, since the observation of a large population of identical systems during a long period is often impossible.

The second approach for failure mode characterization is mainly based on fault injection techniques. Fault injection experiments enable the robustness of the target component to be analyzed from different viewpoints, depending on the way the target component is corrupted. This approach can trigger the system's error detection mechanisms more frequently and also observe how it behaves when the error detection coverage is not $100 \%$, as is usually the case for complex systems. 


\subsection{Fault Injection}

Fault injection is a well-known dependability characterization technique [3], which studies a system's reaction to abnormal conditions. It is a testing approach that is complementary to analytical approaches, and which allows the examination of system states which would not be reached by conventional functional testing. The aim of fault injection is to simulate the effect of real faults impacting a target system, namely the error due to the activation of a fault.

A number of fault injection techniques have been developed. Physical fault injection (e.g., heavy-ion and electromagnetic perturbations, pin-level fault injection) was the initial type of technique used (stuck-at-1, stuck-at-0, bit-flips) [4]. Due to the complexity and the speed of modern integrated circuits, SoftwareImplemented Fault Injection (SWIFI) techniques are often preferred. In this technique, the corruption is performed by a software tool, and can target different components or different layers in a system (operating system kernel, system services, middleware components). This approach is very generic and flexible, since a large variety of fault models can be used. Several studies have shown that a single bit-flip leads to similar errors to those produced by physical fault injection techniques (e.g., 56]), and also that they simulate errors produced by software faults 7] fairly faithfully.

The target for the fault injection can either be the interface of a software component, or its internal address space. Targeting the interface assesses the component's robustness, its ability to function correctly in the presence of invalid inputs and stressful environmental conditions. It is a useful way of evaluating the probability of error propagation from one system component to another. Targeting the address space assesses the impact on the component's behaviour of internal corruptions, resulting either from physical faults or a software faults.

An ideal component would be able immediately to detect the effects of a fault. Its internal error detection mechanisms (such as behavioural checks and executable assertions) would detect the incorrect inputs or the perturbation of the component's state or behaviour, and signal the problem to interacting components. In practice, this is not always the case, particularly with off-the-shelf components where dependability may not be an important criterion. The outputs of a fault injection campaign are thus interesting to (i) discover weak or missing error detection mechanisms and to (ii) identify the unexpected behaviour that must be taken into account at upper layers of the system. Experimental failure mode analysis is thus an input for the design of error confinement wrappers and fault tolerance mechanisms. This last statement shows the important place of fault injection experiments in the design and the implementation of fault tolerant systems.

Several factors must be considered before launching a fault injection campaign:

- the fault model: which classes of errors to insert, where to insert them, and when? The injection may be triggered by the occurrence of an event of interest, or occur after a predetermined time period. The fault may be transient in nature (e.g, a single bit-flip), or permanent (e.g., a bit remains stuck at zero). 
- the observations: how to detect and classify the failure modes? This can be delicate in a distributed system, where failures can be partial.

- the workload: what operational profile or simulated system activity should be applied during the experiment?

There is a considerable body of work applying fault injection for the characterization of operating systems (such as the Ballista work on robustness testing of POSIX-compliant operating systems 8]), and real-time kernels (such as 9, which assesses the behaviour of real-time microkernels in the presence of both external and internal faults). Fault injection has also been applied to network protocol stacks, for example [10] on the characterization of TCP stacks in Unix operating systems. However, there has been little published work targeting middleware. In the next section we describe the methodology we have followed for our fault injection experiments targeting the CORBA Name Service.

\section{Methodology}

In this section, we present the experimental target that we have chosen for our fault injection experiments, and describe the fault model that we have used. We then explain the classification of failure modes that we have chosen, and describe our experimental setup.

\subsection{Experimental Target}

Our dependability characterization work has so far concentrated on middleware services, specifically the CORBA Name Service [11. This service provides a hierarchical directory for object references, allowing server applications to register a service under a symbolic name, and clients to obtain references by resolving a name.

We chose this target since its standardized interface makes it easy to compare different implementations of the service. Furthermore, the Name Service may constitute a single point of failure in a CORBA-based system: while it is possible to deploy applications without using a naming or trading service, by allocating object references statically, most systems require the dynamicity provided by this service.

We believe that the failure modes exhibited by a vendor's implementation of the name service will also be present, to a significant extent, in other applications built using the vendor's CORBA ORB. Indeed, a vendor's name service implementation is typically composed of a certain amount of application code implementing the service-specific functionality, which is linked with the vendor's shared library implementing its ORB. A significant proportion of the robustness failings we have observed are relatively low level, and thus more likely to come from the ORB library than from the application code; we would therefore expect that they will also be present in other applications using the ORB. 


\subsection{Fault Model}

Middleware-based systems are more complicated from an architectural point of view than centralized systems, and are consequently exposed to a wider range of classes of faults:

- physical faults affecting RAM or the processor's registers (so-called Single Event Upsets or soft errors [12]), for example bitflips due to cosmic radiation.

- software faults (design or programming errors) at the application, middleware and operating system levels. For instance, an application may pass a NULL pointer to the middleware, or the middleware may omit checking of error codes returned by the operating system. Previous work described in Section 5 has investigated the impact of this class of faults.

- "environmental" faults, such as the interruption of network connections and disk-full conditions. Exhaustion of resources, such as RAM (due to memory management problems, common in CORBA applications) and file descriptors, arises from "process aging". These conditions will generally be signalled to the middleware by the operating system; a robust implementation should propagate the condition gracefully to the application level, typically by raising an exception.

- communication errors, such as message loss, duplication, reordering or corruption. While this class of fault is widely assumed not to affect middleware that builds on a reliable network transport protocol, as is the case of CORBA's IIOP, recent research discussed below suggests that these classes of error deserve attention.

Our work investigates the impact of the last category of faults: corrupt method invocations arriving over the network. Our experiments consist of sending a corrupted request to the target, and observing its behaviour. This fault model simulates three different classes of faults:

- transient physical faults in the communication subsystem, resulting for example from faulty memory banks in routers, or faulty DMA transfers with the network interface card.

Network corruption, even over reliable transport protocols such as TCP (on which IIOP is based), is more frequent than is commonly assumed. Based on analysis of traffic traces on a LAN and the Internet, 13 reports that approximately one packet in 32000 fails the TCP checksum, and that between one in a few millions and one in 10 billion packets are delivered corrupted to the application level. This is because the 16-bit checksum used in TCP is not able to detect certain errors. While this proportion is very small, it is non-negligible given the high capacity of modern LANs.

- propagation to the target of a fault that occurred on a remote machine interacting with the target. The fault may have affected the remote operating system kernel, its protocol stack implementation, or the remote ORB, leading to the emission of a corrupted request. 
- malicious faults, such as denial of service attacks against the target 1 . Given the pivotal rôle of the name service in most CORBA-based systems, an attacker who can crash the service may be able to cause the entire system to fail. We note, however, that most CORBA systems will be deployed on private networks where all parties can be assumed to be trustworthy.

A general characteristic of fault injection is that the errors which are provoked can simulate the effects of various types of faults: physical, design and implementation, which affect different parts of the system. Different faults often lead to similar classes of errors. We wish to reproduce these errors to assess their impact at the middleware level.

The types of errors we have investigated are single bitflips and the zeroing of two successive bytes in a message. These are among the most common patterns of corruption identified in [13], and we presume that they are representative of error propagation from remote machines.

There are several possible means of injecting these faults. We could use dedicated network hardware, but this is cumbersome and expensive. Using softwareimplemented fault injection, we could inject faults at the protocol transport layer (for example by instrumenting the operating system's network stack, as in [14]). However, this form of corruption has a very high probability of being detected by the remote host's network stack, and therefore of not being delivered to the middleware. Consequently, we choose to inject the fault at the application level, before the data is encapsulated by the transport layer. In this way we simulate the proportion of corrupt packets that TCP incorrectly delivers as being valid.

\subsection{Failure Modes}

Although the classification of failure modes may depend on the target component, the various possible outcomes of a component's behaviour in the presence of faults are similar. Roughly speaking, either the fault is successfully detected by various error detection mechanisms (behavioural checks, executable assertions, hardware mechanisms, etc.) and signalled by different means (error status, exceptions, interrupts, etc.) to the interacting components, or it is not.

The latter case is the more difficult to classify. The first possible situation is the crash or the hang of the target component. Observing this situation involves external mechanisms that control the liveness of the component under test. When no crash or hang are observed, then more subtle mechanisms must be used to distinguish the correct outcomes of the target. In testing, this is known as the notion of oracle. This oracle must be defined beforehand and is part of both the activation profile of the component under test and the fault injection campaign at runtime. Indeed, during a test experiment, the outputs of the component must

\footnotetext{
${ }^{1}$ However, our work is not a realistic study of malicious faults. The errors we simulate in our experiments are unlikely to be representative of the activities of a malicious user of the system, who is likely to employ more sophisticated sequences of unexpected inputs than single bitflips. Robustness is a necessary, but not sufficient, characteristic of survivable systems.
} 
be obtained to be compared (at the end) to the oracle. This is the only way to detect incorrect behaviour of the target component during the test phase, when built-in error detection mechanisms fail.

We classify the experimental outcomes for injections targeting the name service as follows:

- Kernel crash: the machine hosting the service becomes inaccessible from the network. We test for this condition by attempting to execute a command from a remote machine.

- Service crash: attempts to establish a network connection to the service are refused. Typically this means that the process implementing the service has died.

- Service hang: the service accepts the incoming connection, but does not reply within a given time span. Note that this does not necessarily mean that other clients of the service are blocked, since processing may continue in other threads.

- Application failure (error propagation to the application level): the service starts returning erroneous results to clients. We assume conservatively that error propagation to the application causes an application failure.

- Exception: an invocation of the service results in a CORBA exception being raised. We distinguish between System Exceptions (which come from the ORB) and User Exceptions (which are raised at the application level).

These failure modes are not exclusive: for example a service crash will generally result in clients of the service receiving an exception indicating that a communication error has occurred. In the results we provide below, each experiment is classified according to the most serious failure mode observed by the testbed.

The observation of these failure modes is a crucial issue in a fault injection campaign. It is difficult to achieve $100 \%$ coverage of the error detection mechanisms, so some failures may be undetected. In particular, since all fault injection experiments are finite in time, it is possible for an injected fault not to lead to any observable effect during the duration of the experiment. This does not necessarily mean that the fault has no impact, since its effect may be postponed (notion of error latency).

These failure modes are not equivalent from a dependability point of view. Signalling an exception is the "best" experimental outcome, since the service remains available to other users, and the application can decide on the most appropriate recovery action, such as retrying the operation (in the case of a TRANSIENT exception) or deciding to use an alternative service (for COMM_FAILURE). It is important that the exception provide as much information as possible: COMM_FAILURE is more useful than UNKNOWN, since in the latter case the application has less information on which to base its recovery strategy.

The most serious failure mode is error propagation to the application level: indeed, any fault tolerance mechanisms implemented at the application level will not be activated, and the error is free to propagate to the system's service interface. The kernel and service crash, and service hang failure modes, while 
not positive outcomes, are considered less serious, since they can be detected and masked by system-dependent mechanisms such as watchdog timers.

\subsection{Experimental Setup}

The infrastructure we use to support our fault injection experiments consists of the following components:

- the workload application, which activates the target service's functionality (the workload runs on a different machine from the service);

- the fault injector, which sends a corrupted request to the target once the workload has been running for a certain time span;

- monitoring components, which observe the behaviour of the target and log their observations to an SQL database;

- offline data analysis tools.

Our workload application repeatedly constructs a naming graph, resolves names against this graph, and then destroys the graph. Since the graph is built up in a deterministic way, the workload is able to check that the results returned by the service are correct (it plays the rôle of oracle with respect to the functional specification of the service). If the workload detects an anomaly in the operation of the target service, such as an incorrect result, this is signalled as an application failure. If it receives an exception from the target, it signals the appropriate exception outcome.

Each experiment corresponds to a single injected fault. A controller process launches the target service and obtains its object reference (in the implementations which we have targeted, the name service is implemented as a Unix dæmon). It then starts the workload application, passing it the service's reference. After 20 seconds, the fault injector sends a corrupted resolve request to the target service (for a name which has not been given a binding) and waits for the reply. The expected reply is a NotFound exception raised by the naming service. If no reply arrives within 20 seconds, a ServiceHang failure mode is signalled. At the end of the experiment, the monitoring components check for the presence of the different failure modes by trying to launch a command on the target host, checking for returned exceptions, etc.

For each target implementation, a fault injection campaign involves running an experiment for each bit or byte position in the resolve request. A campaign lasts around 48 hours per target for the bitflip fault model.

\section{Experimental Results}

\subsection{Target Implementations}

We have carried out our experiments on four implementations of the CORBA Name Service:

- omniORB 2.8, by AT\&T Laboratories, Cambridge. Freely available under the GNU General Public Licence. Implemented in $\mathrm{C}++$. 
- ORBit 0.5.0, also available under the GNU General Public Licence, and implemented in $\mathrm{C}$.

- ORBacus 4.0.4, a commercial product from Object Oriented Concepts, implemented in $\mathrm{C}++$.

- the tnameserv bundled with version 1.3 of Sun's Java SDK.

All experiments were carried out on workstations running the Solaris 2.7 operating system, connected by a $100 \mathrm{Mb} / \mathrm{s}$ Ethernet LAN. While we tried to make the experimental conditions as similar as possible across experiments, a number of factors require particular attention:

- persistency: the omniORB implementation maintains log files so as to provide persistency across service shutdowns. To ensure a fresh environment for each experiment, we erase these log files before starting the service. The ORBacus implementation can be configured to use log files, but we do not enable them in our experiments. The two other tested implementations do not support persistency.

- number of experiments: as mentioned earlier, we perform experiments for each bit or byte position in the corrupted method invocation. CORBA method invocations contain an ORB-dependent parameter called the service context (which can be used to propagate implementation-specific data and implicitly propagate transactions). The size of this parameter differs slightly between ORB implementations, so the exact number of experiments changes slightly from target to target.

- the ORBit implementation defaults to using non-interoperable object references. We configured it to use standard IIOP profiles.

- we do not reboot the machines after each experiment (this is justified by the fact that we have not observed any host crashes).

In certain experiments, we observe several failure modes: for example a SystemException coupled with a ServiceCrash. In the figures presented below, the failure modes are classified according to gravity, and for each experiment the most serious mode is selected.

\subsection{Analysis of Results}

In this section we present the results of our fault injection experiments, for both the double-zero and bitflip fault models. More general analysis from a dependable system integrator's perspective is presented in Section 6 .

Figure 1 compares the experimental outcomes for each target implementation, for the double-zero fault model. The outcomes whose names in the legend are in capital letters correspond to CORBA SystemExceptions. The NotFound outcome is a CORBA application-level exception raised by the naming service when it cannot resolve a name; this is the expected behaviour of the service for our experiments. The sum of the vertical bars for each target is $100 \%$.

A first remark is that we have not observed any cases of error propagation to the application level, which is a positive point. However, there are a relatively large proportion of service hangs and crashes. 


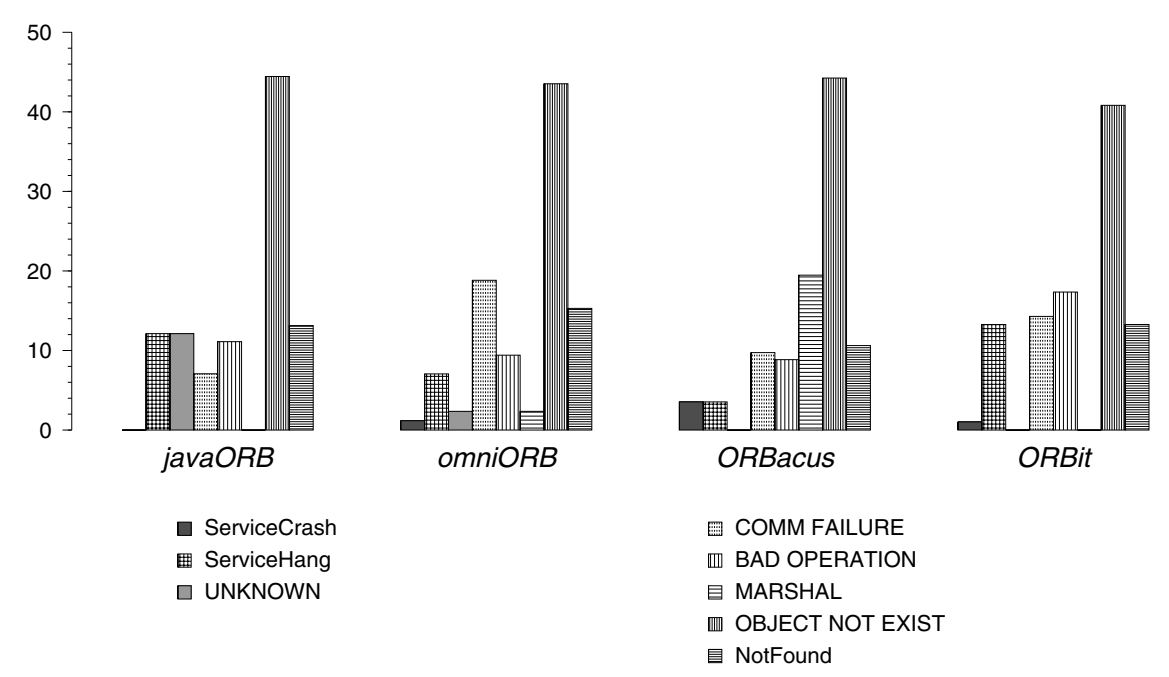

Fig. 1. Experimental outcomes for double-zero fault model

As stated earlier, our service hang failure mode does not imply that other clients of the naming service are blocked; we only consider the time taken to reply to the corrupted invocation. However, given its relative frequency, it is one of the more serious dependability problems we have identified. The upcoming CORBA 2.4 specification allows clients to specify timeouts on their requests, which would be helpful for detecting this type of situation without resorting to applicationlevel watchdog mechanisms. Some of the implementations tested already support these interfaces or provide similar mechanisms.

Examining the details of the breakdown of CORBA exceptions, we observe that the Java implementation raises very few COMM_FAILURE exceptions, but a larger proportion of UNKNOWN exceptions (this exception is raised by an ORB when it detects an error in the server execution whose cause it cannot determine - for example, in Java, an attempt to dereference a null pointer). UNKNOWN is a less useful exception to signal to the application layer, since it conveys no information on the cause of the exception, so from this point of view the Java ORB can be considered less robust. The ORBacus service raises a greater proportion of MARSHAL exceptions, which indicates that its marshalling code does more error checking than other implementations (a positive point from a robustness point of view); ORBit does not raise MARSHAL exceptions.

The proportion of OBJECT_NOT_EXIST exceptions, which the ORB uses to signal that the object reference against which the method was invoked does not exist, is very similar between implementations. This is to be expected, since an ORB is required to check the validity of this value before dispatching the method invocation. A similar remark can be made for the BAD_OPERATION exception. 


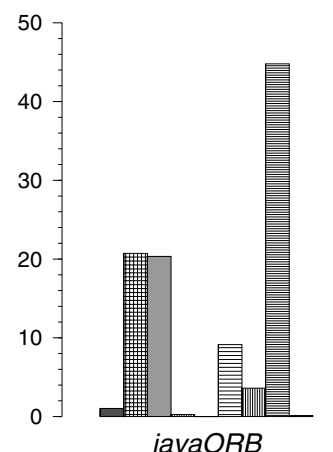

$\square$ ServiceCrash 四 ServiceHang $\square$ UNKNOWN

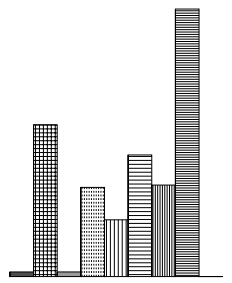

omniORB

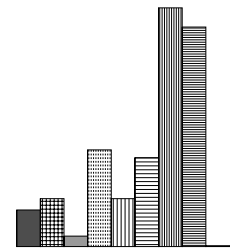

ORBacus

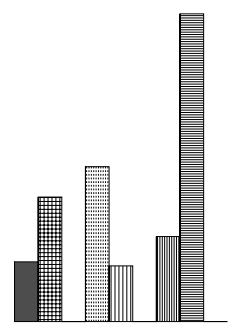

ORBit

$$
\begin{aligned}
& \text { 罭 COMM FAILURE } \\
& \text { 血 BAD OPERATION } \\
& \text { 目 MARSHAL } \\
& \text { 血 OBJECT NOT EXIST } \\
& \text { 自 NotFound }
\end{aligned}
$$

Fig. 2. Experimental outcomes for bitflip fault model

Influence of the error position. Figures 1 and 2 aggregate the results of faults injected at each possible position in the message. It is also interesting to examine the failure modes as a function of the position in the message where the fault was injected. For example, when the fault affects the part of the message which identifies the invoked operation, primarily BAD_OPERATION exceptions are signalled, as would be expected. Similarly, faults injected in the first few bytes of the IIOP request (which contain a special signature which identifies the message type) result mainly in COMM_FAILURE exceptions.

When the fault affects the header bits encoding the message's length, we mostly observe service hangs. Given that there are 32 bits to encode the message length, and that our messages are relatively short (around 900 bits), a bitflip in this zone is likely to increase the announced message length, so the service waits to read more data than will actually arrive.

Differences between fault models. Figure 2 shows the experimental outcomes for each target implementation for the bitflip fault model. The results differ slightly from those for the double-zero fault model. The first difference between the results from the two fault models is the appearance of a InvalidName exception which is not provoked by the double-zero fault model. This exception is raised by the naming service either when the name it is asked to resolve is empty, or -more likely in our case- when the name contains an invalid character.

A second observation is that the bitflip fault model results in a greater proportion of MARSHAL and NotFound exceptions. In the latter case, the difference is likely to be due to the service masking certain errors. Indeed, certain bits 
in an IIOP message are unused. For example, the byte order of a message is represented by a zero or a one marshalled into an octet; seven of these bits are not significant, and so their corruption may not be detected by the ORB. In contrast, a double-zero error is unlikely to escape the notice of the ORB.

Certain other phenomena, such as the small proportion of COMM_FAILURE and BAD_OPERATION exceptions raised by JavaORB for the bitflip fault model, are more difficult to explain.

Internal error checking mechanisms. The ORBacus service was compiled in its default configuration, without disactivating internal "can't happen" assertions. When these assertions fail, the program voluntarily exits using the abort procedure. This leads to ORBacus showing a relatively high proportion of service failures, some of which could be avoided by using a different configuration. The omniORB implementation can be configured at runtime to abort when it detects an internal error, but we did not enable this feature.

System call trace analysis. Our testbed allows us to obtain system call traces and execution stack backtraces of the target process. These show that different middleware implementations activate the operating system in different ways. For instance, the ORBacus implementation makes a large number of 1wp_mutex and lwp_sema calls, which enable the synchronization of threads, whereas the omniORB implementation uses a much narrower range of system calls, primarily for reading and writing to the network and to its log file.

The system call traces also illustrate differences in the level of internal error checking between ORB implementations. For example, when faults are injected into certain bit positions, the ORBit implementation causes a segmentation violation while decoding the corrupted message, and is forcibly aborted by the operating system. In contrast, the ORBacus implementation sometimes detects the corruption internally, and is able to print a warning message indicating the position in the program where the error was detected, before voluntarily aborting. This lack of internal error checking is a reasonable implementation decision for ORBit, since its primary design goals are high performance and a small footprint.

\section{Related Work}

There has been little research on middleware dependability characterization. Indeed, we know of no quantitative data on the failure rates of middlewarebased systems, whereas this topic has been extensively studied for mainframetype applications.

The most interesting work in middleware failure mode analysis through fault injection is 15, which reports on robustness testing of a number of CORBA implementations with respect to corrupted invocations of a portion of the client-side interface exposed by an ORB. For example, the object_to_string operation, which converts an object reference into a textual representation, is invoked with 
an invalid object reference, to see whether the ORB crashes or hangs or signals an exception. This work has only targeted client-side operations; activity that involves interaction between a client and a server is not covered.

The Ballista methodology has also been applied to embedded automotive software built on CAN (Control Area Network), injecting faults such as message loss and reception delays 16 .

There has been some work 17] comparing the robustness of CORBA and DCOM applications with respect to high-level failures. The faults considered are hangs and crashes of threads, processes and machines. Similarly to our results, the authors found a significant proportion of application hangs, which led them to recommend the use of application-level watchdog mechanisms. [18] used fault injection to evaluate the ability of fault tolerance middleware (Microsoft Cluster Server and NT-SwiFT from Bell Labs, which monitor processes and restart them upon failure) to improve the reliability of web and SQL services.

We are not aware of other characterization work on CORBA using fault injection. Other validation efforts have used a functional testing approach (such as the CORVAL project, which aims to test the functional correctness and the interoperability of ORB implementations) or have concentrated on performance evaluation (e.g., [19]).

\section{$6 \quad$ First Lessons Learned}

The experimental results presented in Section 4 show a relatively large variability of behaviour of the target candidates in the presence of faults. This demonstrates that, although the service's interface is standardized, a particular candidate's behaviour depends on the design and implementation decisions made by the vendor. In this section, we adopt the viewpoint of a system integrator who must select a candidate implementation for a safety critical system.

As such, we rank first candidates that deliver significant error reporting information, i.e., those which exhibit fewer service hangs and UNKNOWN exceptions. These are the most problematic failure modes when deciding on fault tolerance strategies and error recovery mechanisms that can meet the system's dependability requirements.

By grouping all the exceptions except for UNKNOWN together, we obtain the percentages for the bitflip fault model shown in Table 1.

Table 1. Ranking of service implementations

\begin{tabular}{|c|c|c|c|c|}
\hline Implementation & Exception & UNKNOWN & Service Hang & Service Crash \\
\hline ORBacus & 88.0 & 1.3 & 6.1 & 4.6 \\
omniORB & 79.5 & 0.6 & 19.3 & 0.6 \\
ORBit & 76.6 & 0.0 & 15.8 & 7.6 \\
Java SDK & 58.0 & 20.3 & 20.7 & 1.0 \\
\hline
\end{tabular}


From this viewpoint, the ORBacus and omniORB implementations exhibit the safest behaviour: more significant exceptions are reported, i.e., fewer UNKNOWN exceptions, and there is a smaller proportion of service hangs. ORBacus has a relatively high rate of service failure, which (as discussed in Section 4.2) is partly due to the configuration we chose. This type of reaction to abnormal situations is not necessarily a negative point from a dependability viewpoint. Many fault tolerance strategies, particularly in a distributed computing context, make a fail silence assumption, which requires components to produce either correct results, or none. Silent failures can successfully be handled by replication, either by using identical copies located on different sites, to deal with physical or environmental faults [20, or by using diversified copies to protect against software faults 21].

We also observed in the experiments that the behaviour depends on the fault model. The results obtained with double zeroing and bitflips lead to a different statistical distribution of the failure modes. However, the resulting numbers do not disturb the ranking given in Table 1. Many issues can influence the observed results. Nevertheless both types of experiments leading to the same conclusions reinforce the confidence one can have in the ranking.

Clearly, many other aspects of middleware dependability must be taken into account in the final selection of a candidate. In particular, the effects of other classes of faults need to be investigated. From this viewpoint, the work done by the Ballista project [15], which uses a different fault model and targets a different part of the middleware, is complementary to ours.

\section{Conclusions and Future Work}

This paper has presented an experimental robustness evaluation method for CORBA-based services, and results of experiments targeting four implementations of the CORBA Name Service. These experiments evaluate the effect of corrupted method invocations at the middleware level.

The implementations we have tested show a non-negligible number of robustness weaknesses, but we have not observed cases of error propagation from the middleware to the application level. Our results suggest that the robustness of CORBA-based systems would be enhanced by the addition of an applicationlevel checksum to GIOP. The achieved failure mode characterization aids in the selection of a candidate middleware implementation for critical systems, and helps system integrators decide on the error detection and recovery mechanisms, fault tolerance strategies and architectural solutions that are needed to meet dependability requirements.

Our technique is non-intrusive, and (thanks to the transparency provided by CORBA) easy to port, both to new implementations of the service, and to alternative operating environments (operating system, hardware platform). The approach could also be applied to assess the robustness of other CORBA services, by modifying the workload and the fault injector.

We plan to continue this work in several directions. Our fault injection tools will be improved to obtain measures of error detection latencies. We plan to 
investigate the influence of the operating system on service behaviour in the presence of faults, by carrying out experiments on Windows2000 and Linux. We would also like to examine the influence of system and network load on the service's failure modes, since previous research on fault injection has shown that error propagation is more likely under heavy load. More generally, we plan to conduct further fault injection experiments using other fault models, including RAM bitflips to simulate the effect of transient hardware faults, and code mutation techniques to study the effect of software faults.

Acknowledgements. This work is partially supported by the European Community (Project IST-1999-11585: DSoS - Dependable Systems of Systems). The authors would like to thank Jean Arlat for helpful comments on their experiments.

\section{References}

1. J. C. Laprie, "Dependable computing: Concepts, limits, challenges," in 25th IEEE International Symposium on Fault-Tolerant Computing - Special Issue, pp. 42-54, IEEE Computer Society Press, 1995.

2. M. Kalyanakrishnam, Z. Kalbarczyk, and R. Iyer, "Failure data analysis of a LAN of Windows NT based computers," in Proceedings of the 18th IEEE Symposium on Reliable Distributed Systems (SRDS '99), (Washington - Brussels - Tokyo), pp. 178-189, IEEE, Oct. 1999.

3. J. Arlat, A. Costes, Y. Crouzet, J.-C. Laprie, and D. Powell, "Fault injection and dependability evaluation of fault-tolerant systems," IEEE Transactions on Computers, vol. 42, pp. 913-923, Aug. 1993.

4. J. Karlsson, P. Folkesson, J. Arlat, Y. Crouzet, G. Leber, and J. Reisinger, "Application of three physical fault injection techniques to the experimental assessment of the MARS architecture," in Proc. 5th IFIP Working Conference on Dependable Computing for Critical Applications: DCCA-6, pp. 267-287, IEEE Computer Society Press, 1998.

5. M. Rimén, J. Ohlsson, and J. Torin, "On microprocessor error behavior modeling," in Proceedings of the 24th Annual International Symposium on Fault-Tolerant Computing, (Los Alamitos, CA, USA), pp. 76-85, IEEE Computer Society Press, June 1994.

6. E. Fuchs, "Validating the fail-silence of the MARS architecture," in Proc. 6th IFIP Int. Working Conference on Dependable Computing for Critical Applications: DCCA-6, pp. 225-247, IEEE Computer Society Press, 1998.

7. H. Madeira, D. Costa, and M. Vieira, "On the emulation of software faults by software fault injection," in Proceedings of the International Conference on Dependable Systems and Networks (DSN2000), pp. 417-426, IEEE Computer Society Press, 2000.

8. P. J. Koopman and J. DeVale, "Comparing the robustness of POSIX operating systems," in Proceedings of the 29th Annual International Symposium on FaultTolerant Computing (FTCS-29), (Los Alamitos, CA, USA), pp. 30-37, IEEE Computer Society Press, 1999. 
9. J.-C. Fabre, M. Rodríguez, J. Arlat, F. Salles, and J.-M. Sizun, "Building dependable COTS microkernel-based systems using MAFALDA," in Proceedings of the 2000 Pacific Rim International Symposium on Dependable Computing (PRDC2000), IEEE Computer Society Press, 2000.

10. S. Dawson, F. Jahanian, and T. Mitton, "Experiments on six commercial TCP implementations using a software fault injection tool," Software Practice and Experience, vol. 27, pp. 1385-1410, Dec. 1997.

11. Object Management Group, Inc, "CORBAServices: Common Object Service Specification: Naming Service Specification," Documentation available at www.omg.org, Object Management Group, Feb. 2001.

12. J. F. Ziegler and G. R. Srinivasan, "Preface: Terrestrial cosmic rays and soft errors," IBM Journal of Research and Development, vol. 40, pp. 2-2, Jan. 1996.

13. J. Stone and C. Partridge, "When the CRC and TCP checksum disagree," in Proceedings of the 2000 ACM SIGCOMM Conference, pp. 309-319, 2000.

14. S. Dawson and F. Jahanian, "Probing and fault injection of dependable distributed protocols," The Computer Journal, vol. 38, no. 4, pp. 286-300, 1995.

15. J. Pan, P. Koopman, D. Siewiorek, Y. Huang, R. Gruber, and M. L. Jiang, "Robustness testing and hardening of CORBA ORB implementations," in Proceedings of the International Conference on Dependable Systems and Networks (DSN2001), IEEE, June 2001.

16. P. Koopman, E. Tran, and G. Hendrey, "Toward middleware fault injection for automotive networks," in Proc. 28th Int. Symposium on Fault-Tolerant Computing (FTCS-28), pp. 127-135, IEEE Computer Society Press, June 1998.

17. P. E. Chung, W. Lee, J. Shih, S. Yajnik, and Y. Huang, "Fault-injection experiments for distributed objects," in Proceedings of the International Symposium on Distributed Objects and Applications (IEEE, ed.), 1999.

18. T. Tsai and N. Singh, "Reliability testing of applications on Windows NT," in Proceedings of the International Conference on Dependable Systems and Networks (DSN2000), pp. 427-436, IEEE Computer Society Press, 2000.

19. S. Nimmagadda, C. Liyanaarachchi, A. Gopinath, D. Niehaus, and A. Kaushal, "Performance patterns: Automated scenario based ORB performance evaluation," in Proceedings of the Fifth USENIX Conference on Object-Oriented Technologies and Systems, pp. 15-28, The USENIX Association, 1999.

20. D. Powell, Delta-4: A Generic Architecture for Dependable Distributed Computing. Berlin, Germany: Springer-Verlag, 1991.

21. K. S. Tso and A. Avizienis, "Community error recovery in N-version software: A design study with experimentation," in Proc. 17th Int. Symposium on FaultTolerant Computing (FTCS-17), pp. 127-135, IEEE Computer Society Press, 1987. 